\title{
A Numerical Study of the Effect of Disorder on Optical Conductivity in Inhomogeneous Superconductors
}

\author{
Long He, Jian Sun, Cunjun Yang, Yun Song \\ Department of Physics, Beijing Normal University, Beijing, China \\ Email: yunsong@bnu.edu.cn
}

Received March 20, 2013; revised April 22, 2013; accepted May 20, 2013

Copyright (C) 2013 Long He et al. This is an open access article distributed under the Creative Commons Attribution License, which permits unrestricted use, distribution, and reproduction in any medium, provided the original work is properly cited.

\begin{abstract}
We present the effect of disorder on the optical conductivity of two-dimensional inhomogeneous superconductors by applying the kernel polynomial method to solve the Bogoliubov-de Gennes equations. By means of the lattice size scaling of the generalized inverse participation ratio, we find that the localization length of the quasiparticle decreases significantly with the increase of the disorder strength. Meanwhile, the weak disorder can readily restrain the Drude weight, while the superconducting gap has the tendency to suppress the low-energy optical conductivity. We also employ the Lanczos exact diagonalization method to study the competition between the on-site repulsive interactions and disorder. It is shown that the screening effect of repulsive interactions significantly enhances the Drude weight in the normal phase.
\end{abstract}

Keywords: Inhomogeneous Superconductor; Disorder Effect; Anderson Localization; Kernel Polynomial Method; Lanczos Method

\section{Introduction}

The strong electron correlations are widely accepted as the key to solve the fundamentally important problems of the high-temperature superconductors [1]. Apart from the electron-electron interactions, the effect of disorder is also an essential ingredient of high temperature superconductors, since a certain extent of structure and chemical inhomogeneity will inevitably exist in the experimental samples, bringing about significant influence on both the excitations of the normal state and the superconducting gap (SG) $[2,3]$. To present the effect of disorder in the inhomogeneous superconductors, the self-consistent field method based on the Bogoliubov-de Gennes (BdG) equations has been widely used [4-7].

In this paper, we study an effective tight-binding model of the inhomogeneous superconductor on a square lattice [5]

$$
H=-t \sum_{\langle i j\rangle \sigma} c_{i \sigma}^{\dagger} c_{j \sigma}+\sum_{i \sigma} \varepsilon_{i} c_{i \sigma}^{\dagger} c_{i \sigma}+\sum_{\langle i j\rangle}\left\{\Delta_{i j} c_{i \uparrow}^{\dagger} c_{j \downarrow}^{\dagger}+H . c\right\},
$$

where $c_{i \sigma}\left(c_{i \sigma}^{+}\right)$are the electronic annihilation (creation) operators at sites $i$ with spin $\sigma$ ( $\uparrow$ or $\downarrow$ ), $t$ denote the hopping integrals between nearest neighbor (NN) sites, $\varepsilon_{i}$ present the on-site disorder energies. The su- perconducting order parameters, $\Delta_{i j}=-V\left\langle c_{j \downarrow} c_{i \uparrow}\right\rangle[8]$, can be obtained by

$$
\Delta_{i j}=-V \int_{-E_{c}}^{E_{c}} \operatorname{Im} G_{i j}^{12}(\omega)(1-2 f(\omega)) \mathrm{d} \omega,
$$

where $V$ represent the $\mathrm{NN}$ attractive interactions, $f(\omega)$ is the Fermi-Dirac distribution function, and $G_{i j}^{12}(\omega)=\left\langle c_{j \uparrow}^{+}|G(\omega)| c_{i \downarrow}^{+}\right\rangle$indicate the off-diagonal Green's function of NN sites $i$ and $j$.

We introduce the kernel polynomial method (KPM) [9] to expend the off-diagonal single particle Green's function $\bar{G}_{i j}^{12}(\omega)$ into a series of Chebyshev polynomials of order $M[8,9]$,

$$
\operatorname{Im} \bar{G}_{i j}^{12}(\tilde{\omega})=-\frac{2}{1-\tilde{\omega}^{2}} \sum_{n} a_{n}^{12} g_{n} T_{n}(\tilde{\omega}),
$$

With

$$
a_{n}^{12}(i, j)=\left\langle c_{j \uparrow}^{+}\left|T_{n}(H)\right| c_{i \downarrow}^{+}\right\rangle /\left(1+\delta_{0, n}\right),
$$

where $\tilde{\omega}$ represent the scaled energies within the interval $[-1,1]$, and $T_{n}(H)=\cos [n \arccos (H)]$ denote the Chebyshev polynomials of the first kind, and we introduce the Lorentz kernel $g_{n}=\sinh [\lambda(1-n) / M] / \sinh (\lambda)$ to overcome the Gibbs oscillation.

We define the on-site disorder energies $\varepsilon_{i}$ as random 
variables distributed uniformly between $-W / 2$ and $W / 2$, where $W$ can be regarded as the strength of disorder. In Figure 1, we present the disorder effects on $\Delta_{i j}$. To our surprise, we also find that disorder can strongly enhance the superconducting order in some small local regions. The strong superconducting local regions emerge near the sites with smaller disorder potentials, while the non-superconducting local regions occur at the hills of the disorder potentials, where the local density of states are very weak. When $W=4.0$ and $V=4.0$, we observe very strong fluctuations of $\Delta_{i j}$ with the maximum value $\Delta_{i j}^{\max }=1.93$, which is quite larger than $\Delta_{0}=1.37$ of the homogeneous case. Here the energies are unit of $t$. Our findings are in good agreement with the results obtained in the inhomogeneous s-wave superconductors, where some isolated superconducting islands are found to survive strong disorder [10].

Neglecting the intervalley scatterings, the interactions can introduce metal-insulator transition (MIT) in the two dimensional (2D) electron systems with a large number of degenerated valleys [11]. Therefore, it is a crucial problem to study the delocalization effect of interactions in 2D systems with disorder. We introduce a new approach [12] to do lattice size scaling of the generalized inverse participation ration (RIPR) [13], which is defined as

$$
G_{2}(\omega)=\frac{\sum_{i} \rho\left(r_{i}, \omega\right)^{2}}{\left[\sum_{i} \rho\left(r_{i}, \omega\right)\right]^{2}}
$$

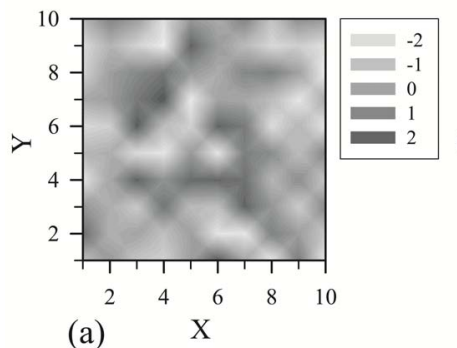

(a)

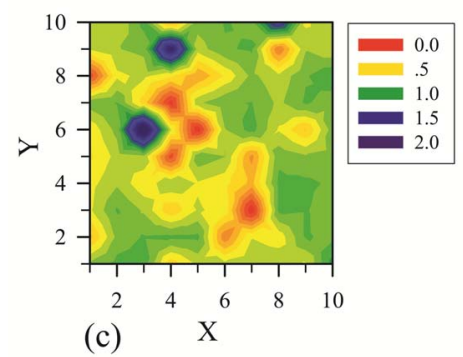

(c)

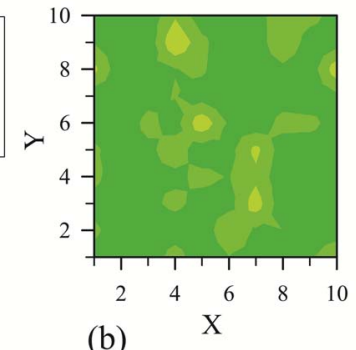

(b)

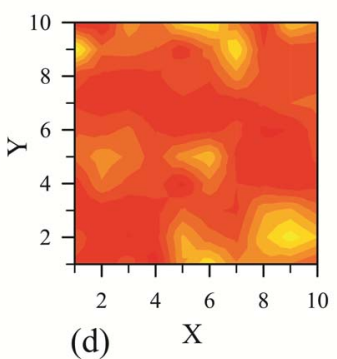

(d) where $\rho\left(r_{i}, \omega\right)$ denote the local density of states (LDOS) at sites $i$. We find that the dependence of $1 / L^{2}$ is a good approximation for the lattice size scaling of GIPR of the 2D inhomogeneous systems. As shown in Figure 2, $G_{2}$ of 2D inhomogeneous superconductors also has very good linear relationship with $1 / L^{2}$, where $L$ represent the size of a square lattice. In addition, it is shown that the intercept on the limitation of $L \rightarrow \infty$ of a localized state increases significantly with the increasing of the disorder strength $W$. Since the localization lengths of quasiparticles are proportion to the the inverse of the square root of the above intercept. Therefore the localization length of quasiparticle decreases with the increase of disorder strength $W$.

The optical conductivity can be calculated by [9]

$$
\sigma(\omega)=\sum_{k, q} \frac{|\langle k|J| q\rangle|^{2}\left[f\left(E_{k}\right)-f\left(E_{q}\right)\right]}{2 Z \omega} \delta\left(\omega-\left(E_{k}-E_{q}\right)\right)
$$

where $J=-i q \sum_{i, \sigma}\left(c_{i, \sigma}^{+} c_{i+1, \sigma}-H . c.\right)$, and $Z$ is the coordination number. In Figure 3(a), we present the effect of disorder on the optical conductivity of the inhomogeneous superconductors with different attractive interactions $V$. We find that the density of optical conductivity in the low-energy region suppressed by the enhancement of superconducting order parameters. In addition, the Drude weight increases slightly with the increase of $V$, suggesting the delocalization effect of $V$, which is in agreement with the prediction drawn from the scaling of GIPR. It is obvious that, in the superconducting phase, the localization effect of disorder is weakened by the off-diagonal superconducting order.

To study the competition between the on-site repulsive interactions and disorder, we employ the Lanczos method [14] to investigate the Hubbard model with box distributed Anderson disorder, which is also called Anderson-Hubbard model with Hamiltonian,

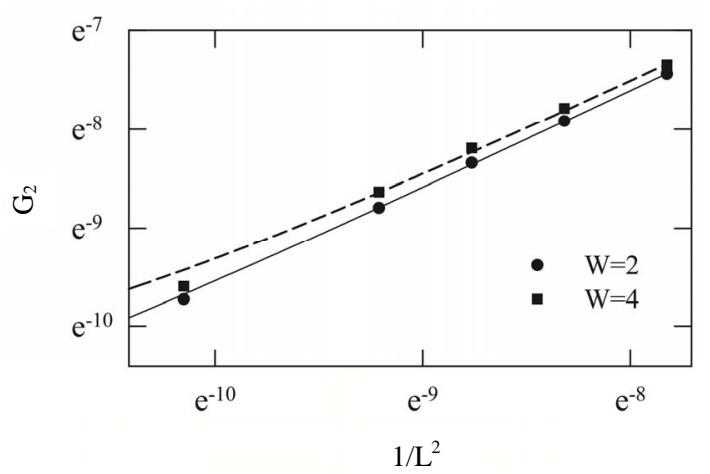

Figure 2. The lattice size scaling of the generalized inverse participation ratio $G_{2}(\omega)$ for the quasiparticles with energy $\omega=1.5 t$ in the conditions with attractive interactions $V=4 t$ and different disorder strength $W$. 


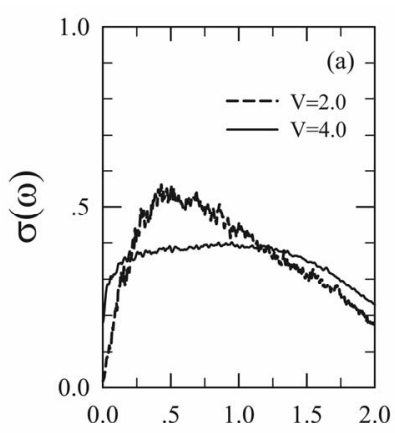

$\omega$

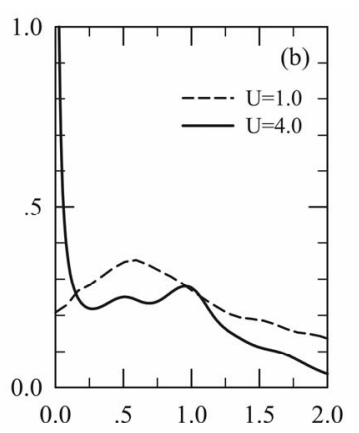

$\omega$
Figure 3. The optical conductivity obtained by (a) the kernel polynomial method for the mean-field superconducting model with $N=200, M=2000$ and $W=4.0$, and by (b) the Lanczos diagonalization method for the Anderson-Hubbard model at half-filling with $N=8$ and $W=4.0$. Energies are in unit of $t$.

$$
H=-t \sum_{\langle i j\rangle \sigma} c_{i \sigma}^{\dagger} c_{j \sigma}+\sum_{i \sigma}\left(\varepsilon_{i}-\mu\right) c_{i \sigma}^{\dagger} c_{i \sigma}+U \sum_{i} n_{i \uparrow} n_{i \downarrow}
$$

where $U$ is the on-site repulsive interactions. We fix the disorder strength $W=4 t$ to study the effect of the on-site repulsive interactions on the optical conductivity. As shown in Figure 3(b), the Drude weight increases significantly when the on-site interactions increase from $U=t$ to $U=4 t$. As discussed in reference [15], the delocalization effect comes from the screening effect of the on-site interaction on the disorder potential.

In summary, the localization effect of disorder has been investigated by applying the scaling of generalized inverse participation ratio. We find that the off-diagonal superconducting order has the delocalization effect, while the on-site repulsive interactions can suppress significantly the localization of quasiparticles by screening strongly the disorder potential. The foregoing solutions can be demonstrated by observing the evolution of Drude weight.

\section{Acknowledgements}

The work was supported by the NSFC of China, under Grant No. 10974018 and 11174036, and the National
Basic Research Program of China (Grant Nos. 2011CBA 00108).

\section{REFERENCES}

[1] P. A. Lee, Reports on Progress in Physics, Vol. 71, 2008, Article ID: 012501. doi:10.1088/0034-4885/71/1/012501

[2] A. N. Pasupathy, A. Pushp, K. K. Gomes, C. V. Parker, J. S. Wen, Z. J. Xu, et al., Science, Vol. 320, 2008, pp. 196-201. doi:10.1126/science. 1154700

[3] K. Chatterjee, M. C. Boyer, W. D. Wise, T. Kondo, T. Takeuchi, H. Ikuta and E. W. Hudson, Nature Physics, Vol. 4, 2008, pp. 108-111. doi:10.1038/nphys835

[4] P. G. de Gennes, "Superconductivity of Metals and Alloys,” Addison-Wesley, Boston, 1989.

[5] W. A. Atkinson, P. J. Hirschfeld and A. H. MacDonald, Physical Review Letters, Vol. 85, 2000, pp. 3922-3925. doi:10.1103/PhysRevLett.85.3922

[6] A. Garg, M. Randeria and N. Trivedi, Nature Physics, Vol. 4, 2008, pp. 762-765. doi:10.1038/nphys1026

[7] H. Alloul, J. Bobroff, M. Gabay and P. J. Hirschfeld, Reviews of Modern Physics, Vol. 81, 2009, pp. 45-108.

[8] L. Covaci, F. M. Peeters and M. Berciu, Physical Review Letters, Vol. 105, 2010, Article ID: 167006. doi:10.1103/PhysRevLett.105.167006

[9] A. Weiße, G. Wellein, A. Alvermann and H. Fehske, Reviews of Modern Physics, Vol. 78, 2006, pp. 275-306. doi: 10.1103/RevModPhys.78.275

[10] A. Ghosal, M. Randeria and N. Trivedi, Physical Review B, Vol. 65, 2001, Article ID: 014501. doi:10.1103/PhysRevB.65.014501

[11] A. Punnoose and A. M. Finkel'stein, Science, Vol. 14, 2005, pp. 289-291. doi:10.1126/science.1115660

[12] Y. Song, H. K. Song and S. P. Feng, Journal of Physics: Condensed Matter, Vol. 23, 2011, Article ID: 205501. doi:10.1088/0953-8984/23/20/205501

[13] N. C. Murphy, R. Wortis and W. A. Atkinson, Physical Review B, Vol. 83, 2011, Article ID: 184206. doi:10.1103/PhysRevB.83.184206

[14] E. Dagotto, Reviews of Modern Physics, Vol. 66, 1994, pp. 763-840. doi:10.1103/RevModPhys.66.763

[15] Y. Song, S. Bulut, R. Wortis and W. A. Atkinson, Journal of Physics: Condensed Matter, Vol. 21, 2011, Article ID: 385601. doi:10.1088/0953-8984/21/38/385601 